OPEN ACCESS

Edited by:

Konstantin V. Korotkov,

University of Kentucky, United States

Reviewed by:

Ashu Sharma,

University at Buffalo, United States Vikram Saini,

University of Alabama at Birmingham, United States

*Correspondence: Xuemei Zhang apoe@163.com

Kaifeng Wu

kiphoonwu@126.com

tThese authors have contributed equally to this work.

Specialty section: This article was submitted to

Infectious Diseases,

a section of the journal

Frontiers in Microbiology

Received: 09 January 2018 Accepted: 16 May 2018

Published: 13 June 2018

Citation: Ye W, Zhang J, Shu Z, Yin Y, Zhang $X$ and Wu K (2018) Pneumococcal LytR

Protein Is Required for the Surface Attachment of Both Capsular Polysaccharide and Teichoic Acids: Essential for Pneumococcal Virulence.

Front. Microbiol. 9:1199.

doi: 10.3389/fmicb.2018.01199

\section{Pneumococcal LytR Protein Is Required for the Surface Attachment of Both Capsular Polysaccharide and Teichoic Acids: Essential for Pneumococcal Virulence}

\author{
Weijie Ye ${ }^{1 t}$, Jinghui Zhang ${ }^{1 \dagger}$, Zhaoche Shu ${ }^{1}$, Yibing Yin ${ }^{1}$, Xuemei Zhang ${ }^{1 *}$ and \\ Kaifeng $W u^{1,2 *}$
}

' Key Laboratory of Diagnostic Medicine Designated by the Ministry of Education, Department of Laboratory Medicine,
Chongqing Medical University, Chongqing, China, ${ }^{2}$ Department of Laboratory Medicine, The Third Affiliated Hospital of Zunyi Medical University, Zunyi, China

The LytR-Cps-Psr family proteins are commonly present in Gram-positive bacteria, which have been shown to implicate in anchoring cell wall-related glycopolymers to the peptidoglycan. Here, we report the cellular function of SPD_1741 (LytR) in Streptococcus pneumoniae and its role in virulence of pneumococci. Pneumococcal $\Delta / y t R$ mutants have been successfully constructed by replacing the lytR gene with erm cassette. The role of LytR in pneumococcal growth was determined by growth experiments, and surface accessibility of the LytR protein was analyzed using flow cytometry. Transmission electron microscopy (TEM) and immunoblotting were used to reveal the changes in capsular polysaccharide (CPS). Dot blot and ELISA were used to quantify the amount of teichoic acids (TAs). The contribution of LytR on bacterial virulence was assessed using in vitro phagocytosis assays and infection experiments. Compared to the wild-type strain, the $\Delta / y t R$ mutant showed a defect in growth which merely grew to a maximal $O D_{620}$ of 0.2 in the liquid medium. The growth of the $\Delta / y t R$ mutant could be restored by addition of recombinant $\Delta T M$-LytR protein in culture medium in a dose-dependent manner. TEM results showed that the D39 $\Delta / y t R$ mutant was impaired in the surface attachment of CPS. Deletion of lytR gene also impaired the retention of TAs on the surface of pneumococci. The reduction of CPS and TAs on the pneumocccal cells were confirmed using Dot blot and ELISA assays. Compared to wildtype D39, the $\Delta / y t R$ mutant was more susceptible to the phagocytosis. Animal studies showed that the ability to colonize the nasophaynx and virulence of pneumococci were affected by impairment of the lytR gene. Collectively, these results suggest that pneumococcal LytR is involved in anchoring both the CPS and TAs to cell wall, which is important for virulence of pneumococci.

Keywords: Streptococcus pneumoniae, IytR, teichoic acids, capsular polysaccharide, virulence 


\section{INTRODUCTION}

Streptococcus pneumoniae (S. pneumoniae) is a well-known human pathogen which, like other Gram-positive bacteria, is commonly decorated with teichoic acids (TAs) and capsular polysaccharide (CPS) on its outer surface of the bacterial body (Skov Sorensen et al., 1988; Hammerschmidt et al., 2005; Weidenmaier and Peschel, 2008). In S. pneumoniae, capsule is recognized as the most important determinant required for bacterial virulence, since less encapsulated or non-encapsulated pneumococcal strains are less virulent or avirulent (Hyams et al., 2010; Wu et al., 2016). TAs are another important glycopolymers which are comprised of cell-wall associated teichoic acid (WTA) and lipoteichoic acid (LTA) (Wu et al., 2014). TAs were implicated in several biological functions, including bacterial growth, bacterial division and morphology, and cellular roles in host-cell interaction (Wu et al., 2014; Liu et al., 2017).

The biosynthesis of both CPS and TAs is a complex process and requires multiple steps in S. pneumoniae. Except for serotypes 3 and 37, it has been indicated that the Wzydependent pathway was responsible for the biosynthesis of CPS (Arrecubieta et al., 1994; Llull et al., 1999). In addition, without counting the transcriptional regulators (Wu et al., 2016; Zheng et al., 2017), there are other proteins including Pgm and GalU which are involving in sugar metabolism determine the CPS biosynthesis (Mollerach et al., 1998; Hardy et al., 2001). As for the biosynthesis of TAs, a previous bioinformatic analysis suggested the involvement of 16 known genes and other hypothetical genes, primarily clustered in the lic loci, in the biosynthesis of pneumococcal TAs (Denapaite et al., 2012). In 2014, we found that RafX was implicated in the ligation of WTA to the cell wall (Wu et al., 2010). Recent study by Liu et al. has revealed that SPD_1198 and SPD_1197 were responsible for the polymerization process of TAs (Liu et al., 2017). Despite current progress in identification of these enzymes, so far, not all the critical enzymes involved in the biosynthesis of both glycopolymers are identified.

Previous work by Kawai et al. has suggested the function of LytR-CpsA-Psr family proteins (LCP) in the attachment of WTA to peptidoglycan in Bacillus subtilis (B. subtilis) (Kawai et al., 2011). Other studies in Staphylococcus aureus (S. aureus) and B. anthracis have also revealed the necessary of LCP enzymes in the attachment of either CPS or TAs to the cell wall (Chan et al., 2013, 2014; Liszewski Zilla et al., 2015). More recently, Gale et al. (2017) and Schaefer et al. (2017) using in vitro enzymatic assays demonstrated independently the ligase activities of LCP in attaching the WTA to the peptidoglycan in S. aureus and B. subtilis. These lines of evidence indicated that LCP proteins have ligase activity which contributed to the attachment of WTA to the cell wall and probably the CPS to the cell wall.

In $S$. pneumoniae, there are three proteins including SPD_1741 (LytR), Cps2A, and SPD_1202 (Psr) belonging to the LCP family. It has been indicated that Cps $2 \mathrm{~A}$ has the phosphotransferase activity and knocking down cps $2 \mathrm{~A}$ reduced the amount of capsule on the cell wall (Eberhardt et al., 2012). Inactivation of both cps $2 A$ and lytR ( $\left.S P D \_1741\right)$ significantly impaired the retention of CPS on the cell wall in S. pneumoniae
D39 (Eberhardt et al., 2012). It is known that knocking down cps $2 \mathrm{~A}$ alone is sufficient to cause obvious loss of CPS, speculating the involvement of LytR in CPS production is indirect and may be not sufficient. While two previous studies have showed that pneumococcal LytR is essential for S. pneumoniae viability (Johnsborg and Havarstein, 2009; Eberhardt et al., 2012), using the CRISPRi knockdown technique, Liu et al. did not prove the lytR as the necessary gene in S. pneumoniae (Liu et al., 2017). These motivate us to further clarify the function of the LytR in S. pneumoniae by preparing single lytR mutant.

In this study, pneumococcal lyt $R$ gene was successfully replaced with $\mathrm{erm}$ cassette to obtain lytR mutants. We found that the $\Delta l y t R$ mutant was impaired in growth, and addition of recombinant LytR protein to the culture medium restored the growth in a dose dependent manner. Using TEM analysis and other immunological analytical methods, we showed that pneumococcal LytR was involved in both the attachment of CPS and TAs to the cell wall. Finally, we showed that the inactivation of LytR may be disastrous to $S$. pneumoniae during infecting the host.

\section{MATERIALS AND METHODS}

\section{Bacterial Strains, Plasmids, Bacterial Growth Conditions}

All of the bacterial strains, plasmids, and primers used in this study are listed in Supplementary Tables S1, S2 in Supplementary Material. E. coli strains were grown in Luria-Bertani (LB) broth with shaking or on LB agar plates at $37^{\circ} \mathrm{C}$. S. pneumoniae strains were grown in semisynthetic casein hydrolysate medium supplemented with $5 \%$ yeast extract $(\mathrm{C}+\mathrm{Y}$ medium, $\mathrm{pH} 7.0$ ) or on blood agar plates at $37^{\circ} \mathrm{C}$ in an atmosphere of $5 \% \mathrm{CO}_{2}$. Bacteria were stored in growth medium supplemented with $10 \%$ glycerol at $-80^{\circ} \mathrm{C}$. Appropriate antibiotics (Supplementary Table S1) were supplemented to bacterial growth medium when needed.

\section{Expression and Purification of the $\Delta T M-L y t R$ Protein}

Since the first 30 amino acid residues belong to the transmembrane domain (TM), to facilitate prokaryotic expression of soluble protein, the $\triangle \mathrm{TM}$-LytR encoding region was amplified from the genome of S. pneumoniae D39 using PCR technology with primer pairs LytR-F and LytR-R (Supplementary Table S2). The resulting lytR product was purified and digested with restriction enzymes, and then cloned into the pW28 plasmid which was further used to transform E. coli BL21 (DE3) competent cell. The E. coli bacteria with $\Delta$ TM-LytR-pW28 plasmid were grown in $500 \mathrm{ml} \mathrm{LB}$ medium supplemented with $50 \mu \mathrm{g} / \mathrm{ml}$ kanamycine for $8 \mathrm{~h}$ in a shaking incubator. Isopropyl $\beta$-D-Thiogalactoside (IPTG) at a concentration of $0.25 \mathrm{mM}$ was used to induce the expression of $\mathrm{r} \Delta \mathrm{TM}$-LytR protein. Bacteria were pelleted at $6000 \mathrm{rpm}$ for $30 \mathrm{~min}$ at $4^{\circ} \mathrm{C}$, resuspended in $25 \mathrm{ml}$ binding buffer containing $100 \mu \mathrm{M}$ PMSF, and then were subjected to sonication. $\mathrm{r} \Delta \mathrm{TM}$-LytR protein was purified using 
$\mathrm{Ni}^{2+}$-NTA sepharose column, its purity was analyzed by SDSPAGE. Aliquots of $\mathrm{r} \Delta \mathrm{TM}-\mathrm{LytR}$ protein were stored at $-80^{\circ} \mathrm{C}$.

\section{Construction of lytR Mutants}

The primers used in this study were listed in Supplementary Table S2. A length of 2467-bp cassette containing SPD_1741 (lytR) upstream and downstream fragments and erm encoding sequence was generated by PCR using the primers $\Delta$ lytRP1, $\Delta$ lytR-P2, $\Delta$ lytR-P3, and $\Delta$ lytR-P4. The resulting fragment was used to transform the $S$. pneumoniae strains. The transformation procedures, colony selection and identification were performed according to established protocols ( $\mathrm{Wu}$ et al., 2010). Erythromycin resistant colonies were selected and the targeted sequences were amplified by PCR and sequenced.

\section{Construction of Complemented Strains}

The plasmid pJWV25 with full-length of lytR gene was used to complement the $\Delta l y t R$ mutants (Eberhardt et al., 2009). The full-length lytR fragment was cloned into plasmid pJWV25 at the Spe I and Not I restriction sites. The resulting recombinant plasmid was used to transform $\Delta l y t R$ mutants and selected by tetracycline to obtain the complemented strains $(\Delta l y t R-\mathrm{C})$. For functional assays, the complemented strain was grown in normal $\mathrm{C}+\mathrm{Y}$ medium or supplemented with $0.15 \mathrm{mM} \mathrm{Zn}^{2+}$.

\section{Growth Curve}

A volume of $10 \mu \mathrm{l}$ of stored wild-type D39 strain and the D39 $\Delta l y t R$ mutant were grown on Columbia blood agar plates for $16 \mathrm{~h}$. Bacteria were harvested from the blood agar plates and washed twice in phosphate-buffered saline (PBS). Bacteria were resuspended to an $\mathrm{OD}_{620}=0.4$. For growth on Columbia blood agar plate, $10 \mu \mathrm{l}$ of bacterial suspensions of wild-type D39 and the $\mathrm{D} 39 \Delta l y t \mathrm{R}$ mutant were separately inoculated onto one Columbia blood agar plate and grown at $37^{\circ} \mathrm{C}$ in an atmosphere of $5 \% \mathrm{CO}_{2}$ for $8 \mathrm{~h}$. For growth experiment in liquid medium, suspensions of $100 \mu \mathrm{l}$ were seeded into $5 \mathrm{ml}$ fresh liquid medium. The wild type D39 and the $\Delta l y t R$ mutant were grown in normal $\mathrm{C}+\mathrm{Y}$ medium. $\mathrm{D} 39 \Delta l y t \mathrm{R}$ mutant was grown in $\mathrm{C}+\mathrm{Y}$ medium with different amounts of $\mathrm{r} \Delta \mathrm{TM}-\mathrm{LytR}(1 \mathrm{ng}, 10 \mathrm{ng}, 100 \mathrm{ng}$ and $500 \mathrm{ng}$, respectively) to evaluate the role of LytR in bacterial growth. $\mathrm{D} 39 \Delta l y t R$ grown in $\mathrm{C}+\mathrm{Y}$ medium with $500 \mathrm{ng}$ pneumolysin (PLY) was used as a negative control. Bacteria were cultured at $37^{\circ} \mathrm{C}$ in an atmosphere of $5 \% \mathrm{CO}_{2}$, and $\mathrm{OD}_{620}$ values were recorded at the indicated times.

\section{Transmission Electron Microscopy (TEM)}

A volume of $10 \mu \mathrm{l}$ of stored wild-type D39 strain and the $\mathrm{D} 39 \Delta l y t R$ mutant were grown on Columbia blood agar plates for $16 \mathrm{~h}$. Bacteria were harvested from the blood agar plates and washed twice in phosphate-buffered saline (PBS). Bacteria were resuspended to an $\mathrm{OD}_{620}=0.4$. A volume of $200 \mu \mathrm{l}$ of bacterial suspension was added to $10 \mathrm{ml}$ fresh $\mathrm{C}+\mathrm{Y}$ medium or $\mathrm{C}+\mathrm{Y}$ medium supplemented with $0.15 \mathrm{mM} \mathrm{ZnCl}_{2}(\Delta l y t R-\mathrm{C})$ and cultured at $37^{\circ} \mathrm{C}$ till an $\mathrm{OD}_{620}=0.15$ for the lytR mutant and an $\mathrm{OD}_{620}=0.4$ for the others. Bacteria were harvested by centrifugation at $10000 \mathrm{rpm}$ for $20 \mathrm{~min}$ at $4^{\circ} \mathrm{C}$. Samples were fixed in $1 \mathrm{ml}$ sodium cacodylate buffer supplemented with $2 \%$ glutaraldehyde $(\mathrm{pH} 7.4)$ at $4^{\circ} \mathrm{C}$ for $16 \mathrm{~h}$ before being subjected to analysis. Other procedures were processed by the Electron Microscopy Research service of Chongqing Medical University.

\section{Analysis of TAs/CPS}

Bacteria were prepared as described for TEM analysis. Bacteria were harvested by centrifugation at $10000 \mathrm{rpm}$ for $5 \mathrm{~min}$. The cells were washed twice in phosphate-buffered saline (PBS), and lysed with $0.5 \%$ sodium deoxycholate solution (DOC) containing PMSF at $37^{\circ} \mathrm{C}$ for $30 \mathrm{~min}$. Cells were observed by optical microscope to ensure complete lysis. Protein levels were determined by Bradford method (Bio-Rad Protein Assay). For standardization, the protein levels in all bacterial lysates were adjusted to a concentration of $4 \mathrm{mg} / \mathrm{ml}$, and the protein levels were adjusted to concentrations of $1 \mathrm{mg} / \mathrm{ml}$ and $3 \mathrm{mg} / \mathrm{ml}$ for culture supernatant of R6 and D39, respectively. The amounts of CPS and TAs were analyzed by Western blot, Dot blot, or enzyme-linked immunosorbent assay (ELISA) according to the established protocols (Wu et al., 2014; Zheng et al., 2017).

\section{Fluorescence Activating Cell Sorter (FACS)}

Bacteria were prepared as described above. The bacterial cells were harvested by centrifugation at 12,000 rpm for $5 \mathrm{~min}$ and washed twice in PBS. $1 \times 10^{6} \mathrm{CFU}$ bacteria were inactivated in water bath at $56^{\circ} \mathrm{C}$ for $30 \mathrm{~min}$, resuspended in $200 \mu \mathrm{l} \mathrm{PBS}$ containing $1 \mu \mathrm{l}$ anti-CWPS or anti-LytR, and incubated at $37^{\circ} \mathrm{C}$ for $60 \mathrm{~min}$. Cells were washed twice with PBS, and probed with $200 \mu \mathrm{l}$ PE-labeled goat anti-rabbit IgG or goat anti-mouse IgA (diluted at 1:200, secondary antibodies). After three washes with PBS, cells were subjected to detection. Samples were analyzed by technicians in Children's Hospital of Chongqing Medical University.

\section{Animal Studies}

6-8-week-old C57BL/6 mice were obtained and raised at the experimental animal center, Chongqing Medical University [certificate no. SYXK(yu) 2007-0001]. All the animal experiments were discussed with and approved by the Animal Care and Use Committee of Chongqing Medical University. All procedures were performed according to the recommendations in the Guide for the Care and Use of Laboratory Animals, and conformed to animal protection laws of China and applicable guidelines.

Bacteria were prepared as described above. The $\Delta l y t R$-C strain used in animal experiments was grown in $\mathrm{C}+\mathrm{Y}$ supplemented with $0.15 \mathrm{mM} \mathrm{ZnCl}_{2}$ to induce the expression of LytR. Groups of 6-week-old male C57BL/6 mice were intranasally challenged with $1 \times 10^{7} \mathrm{CFU}$ of bacteria. Survival of the animals was recorded every day until the end of the observation period (15 days). As for colonization experiments, mice were sacrificed after infection with pneumococci for $24 \mathrm{~h}$ or $48 \mathrm{~h}$. Bacteria was harvested from nasal lavage fluid which was serially diluted and plated on the blood agar plates to have bacterial numbers.

Lungs were inflated with $0.5 \%$ agarose under $25-\mathrm{cm}$ water pressure and fixed in 10\% buffered formalin for 24 hours. 
Next, formalin-fixed, paraffin-embedded 4-mm sections of lungs were used for immunohistochemical analysis with hematoxylin eosinstain (Hoogerwerf et al., 2011; Cao et al., 2014).

\section{Anti-Phagocytosis Assays}

Bacteria were prepared as described above. Density of bacterial suspension was adjusted to $\mathrm{OD}_{620}=0.4 .200 \mu \mathrm{l}$ of bacterial suspension was added to $10 \mathrm{ml}$ fresh $\mathrm{C}+\mathrm{Y}$ medium. The wild-type D39 and $\Delta l y t R-\mathrm{C}$ and the $\Delta l y t R$ grown with $L y t R$ protein were cultured to mid-exponential phase $\left(\mathrm{OD}_{620}=0.4\right)$. The $\Delta l y t R$ mutant and $\Delta l y t R+$ PLY protein were cultured to an $\mathrm{OD}_{620}=0.15$. The bacterial density was adjusted to 1 $\times 10^{7} \mathrm{CFU} / \mathrm{ml}$. Macrophages $\left(1 \times 10^{5}\right.$ cells $)$ derived from mice peritoneal cavity were incubated with bacteria at a MOI 100 at $37^{\circ} \mathrm{C}$ for $30 \mathrm{~min}$ according to previous protocols with modifications (Kodali et al., 2013; Sharif et al., 2013). After three washes with PBS, cells were lysed with $\mathrm{ddH}_{2} \mathrm{O}$ and plated onto blood agar plates. The bacteria numbers were counted and survival rates were calculated.

\section{Statistical Analysis}

Differences between groups were analyzed by two-tailed $t$-test or Mann-Whitney U test using Graph-Pad Prism 5 software. Survival rates were compared using the log-rank (Mantel-Cox) test. A $P$ value of $\leq 0.05$ was considered statistically significant.

\section{RESULTS}

\section{Expression and Purification of $\Delta T M-L y t R$ Protein}

Using online bioinformatic tools, it was found that S. pneumoniae SPD_1741 (LytR) protein was a hydrophilic protein with a relative molecular weight of $37.56 \mathrm{kDa}$ and an iso-electric point of 5.11. S. pneumoniae LytR protein belongs to the LytR_cpsA_psr family and was predicted to be an anionic cell wall polymer synthesis related enzyme. Our attempts to prepare full length LytR protein were unsuccessful. By structural analysis using TMHMM online software (Supplementary Figure S1), it was found that LytR protein contains 1 predicted transmembrane (TM) helices within the first 30 amino acid residues at the $\mathrm{N}$-terminus, and the enzymatic domain was located on the outside the cell membrane. Therefore, only the predicted extracellular domain of LytR protein was overexpressed in E. coli BL21 (DE3). As shown in Figure 1, the recombinant $\Delta$ TMLytR could be generated in soluble forms. Over $90 \%$ purity could be achieved by purification with the $\mathrm{Ni}^{2+}$-NTA affinity chromatography. The purified $\mathrm{r} \Delta \mathrm{TM}$-LytR protein was used to prepare the anti-LytR antiserum and used for following functional assays.

\section{Surface Accessibility of LytR Protein in S. pneumoniae}

TMHMM analysis showed that the enzymatic domain of LytR was located outside the cell membrane (Supplementary Figure S1). In addition, tracing with GFP protein, it has been

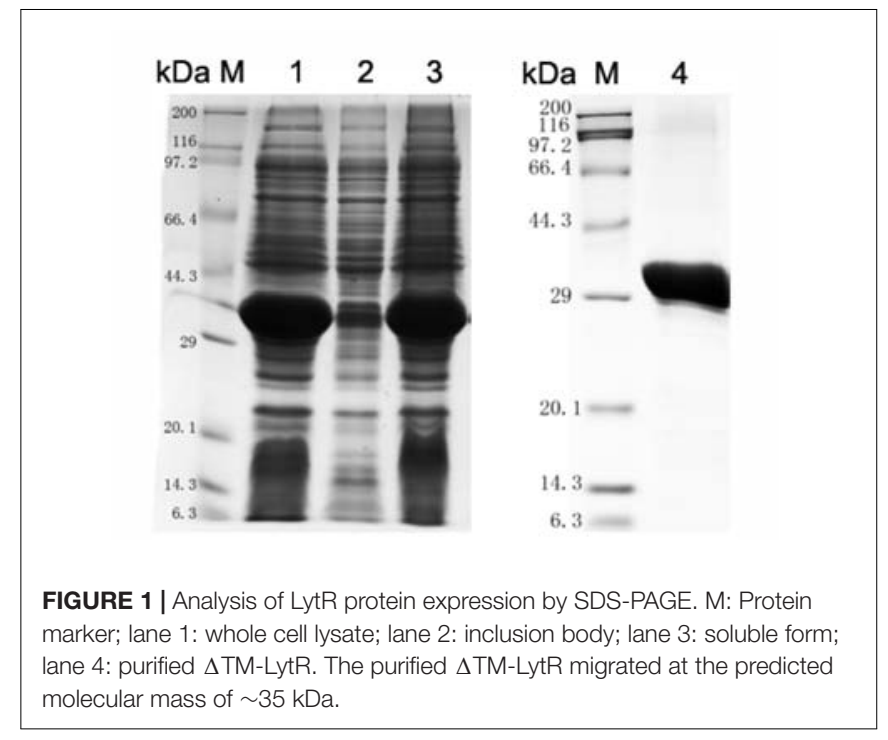

shown that LytR was located in the membrane (Eberhardt et al., 2012). However, it remains unclear whether the enzymatic domain of LytR was exposed outside the bacterial bodies. It has been shown that LytR was expressed throughout the growth phase (Johnsborg and Havarstein, 2009). Therefore, pneumococci grown in the early exponential phase were collected for FACS analysis. Wild-type R6 and $\Delta l y t R$ mutant were incubated with anti-LytR antiserum, and probed with PE-labeled goat anti-mouse secondary antibodies. The surface location of TAs was used as positive control. As shown in Figure 2, it was found that, compared to the $\Delta l y t R$ mutant showing no positive fluorescence signal, the LytR signal in wild-type R6 was obvious. Combined with evidence available, it was strongly suggested that LytR is a membrane protein and its predicted enzymatic domain is surface accessible.

\section{LytR Protein Was Essential for Pneumococcal Growth}

To test the function of $S$. pneumoniae LytR, we created the $\Delta l y t R$ mutant on the genetic background of R6 and D39 in which the lytR gene was replaced with erm cassette using insertiondeletion mutagenesis strategy. The deletion of $l y t R$ gene was proved to be a nonpolar event verified by reverse transcription (RT)-PCR. To further confirm the role of LytR, we complemented the lytR gene ectopically with the pJWV25 plasmid. Sequencing of the respective genome regions confirmed that the mutations or insertions generated as expected.

Inactivation of $l y t R$ led to significant growth defects in either the blood agar plate or the liquid medium. Compared with wildtype D39, the $\Delta l y t R$ mutant was smaller but remained smooth like wild-type D39 (Figure 3A). When growing in the liquid medium, the $\Delta l y t R$ mutant showed significantly reduced growth rate compared to the wild-type strain. The $\mathrm{D} 39 \Delta l y t R$ mutant only grew to a maximal $\mathrm{OD}_{620}$ of 0.2 (Figure 3B).

As shown in Figure 2, since LytR in wild type pneumococci was surface exposed, we wanted to see whether the addition 

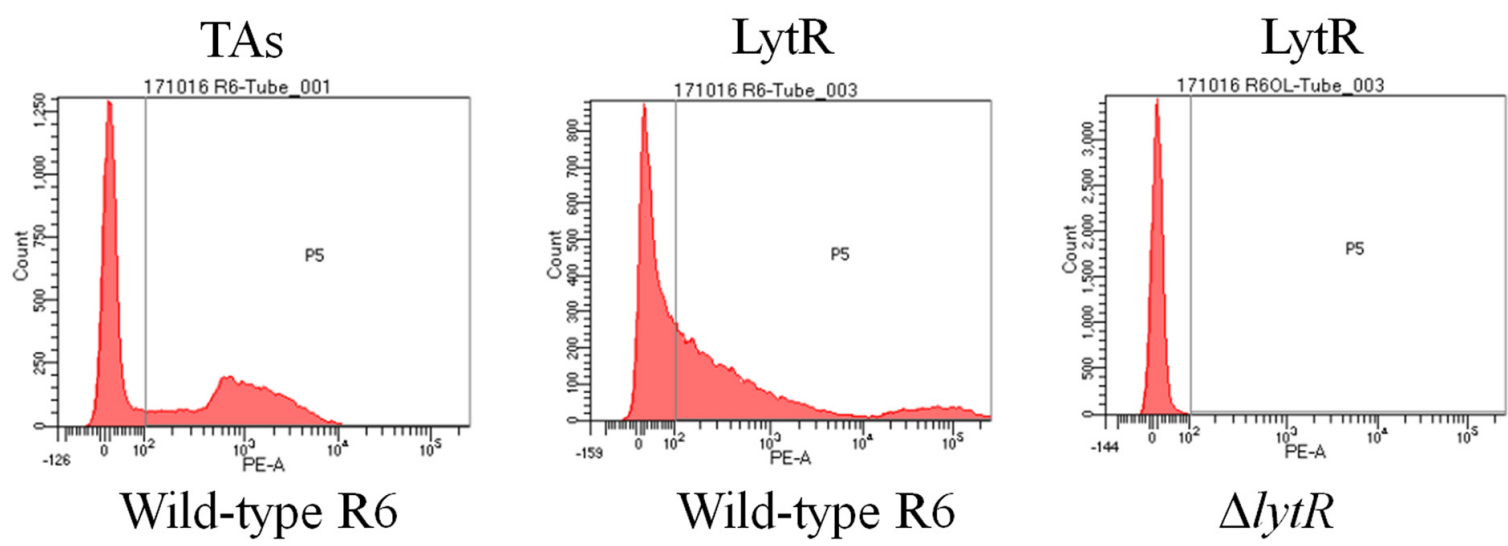

FIGURE 2 | Surface accessibility of pneumococcal LytR. Wild-type R6 (WT) was incubated with either anti-CWPS antiserum (probes TAs) or anti-LytR antiserum and probed with the PE-labeled secondary antibodies. $\Delta / y t R$ mutant was used as negative control. Three independent experiments were performed and representative figures were presented.
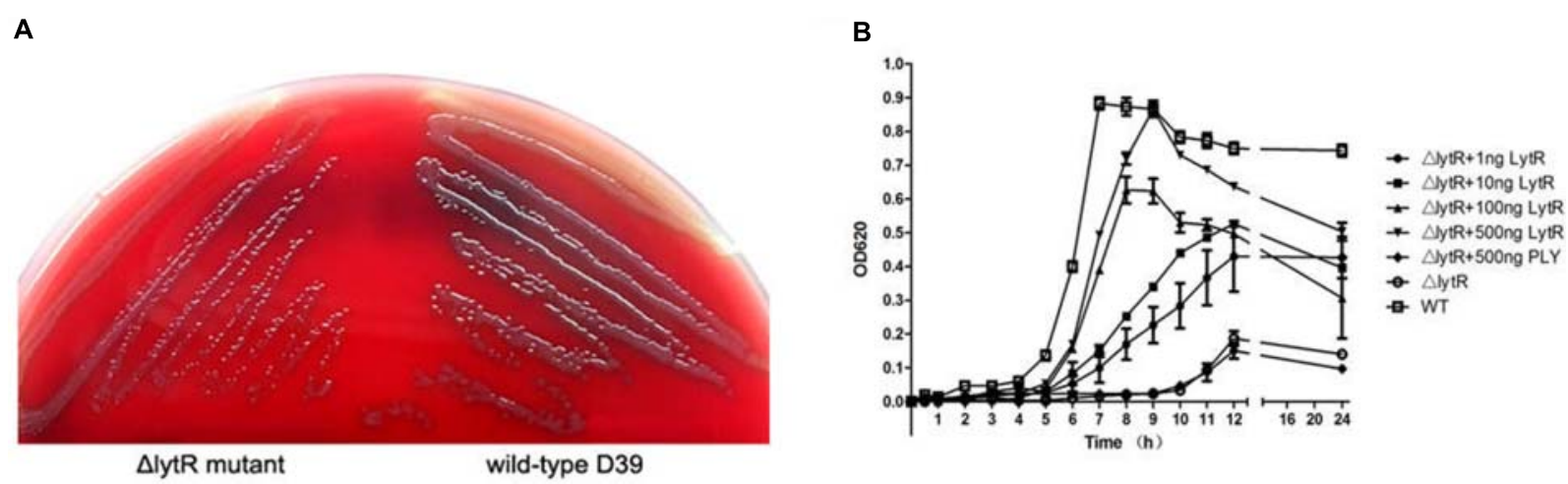

FIGURE 3 | Growth characteristics of the strains. Equal volumes containing equal bacterial numbers of wild-type D39 and the $\Delta / y t R$ mutant were grown on blood agar plates for $8 \mathrm{~h}$ (A). Growth curves of strains grown in $\mathrm{C}+\mathrm{Y}$ medium or medium supplemented with the indicated proteins (B). Cell densities were measured at the indicated time points. The control protein, PLY, not involved in bacterial control was used as control. The values represent means of three independent with SEM.

of the recombinant $\Delta$ TM-LytR could complement the growth defect. $1 \mathrm{ng}, 10 \mathrm{ng}, 100 \mathrm{ng}$, and $500 \mathrm{ng}$ recombinant $\Delta$ TM-LytR proteins were added into the culture medium of $\Delta l y t R$ mutant and the optical densities were measured at $\mathrm{OD}_{620}$, respectively. As a control, $500 \mathrm{ng}$ of the recombinant pneumolysin (Ply) was added into the culture medium of the $\Delta l y t R$ mutant. As shown in Figure $3 B$, the recombinant $\Delta \mathrm{TM}$-LytR improved growth of the $\Delta l y t R$ mutant in a dose-dependent manner, while the addition of Ply was ineffective.

\section{LytR Was Required for Attachment of the Capsular Polysaccharide to Cell Wall}

Since the $\Delta l y t R$ mutant grown on blood agar plate displayed small and smooth colony phenotypes, and considering previous indication regarding the role of LCP family proteins in the biosynthesis of glycopolymers, we analyzed the characteristics of the lytR mutant by TEM (Figure 4). The complemented lytR mutant and the $\Delta l y t R$ mutant grown in the medium supplemented with $500 \mathrm{ng}$ recombinant $\Delta \mathrm{TM}$-LytR protein were analyzed in parallel. The TEM results showed that the thickness of capsule present on the bacterial surface was significantly reduced in D39 $\Delta$ lyt $R$ mutant cells relative to that of the wildtype D39 cells, indicating a defect in CPS biosynthesis. Both the complemented strain $(\Delta l y t R-C)$ and the $\Delta l y t R$ mutant grown in the surrounding with recombinant LytR protein restored the capsule, suggesting the direct involvement LytR in attaching the CPS to the cell wall.

Immnoblotting assays were further performed to determine the production of CPS in lysates of strains. Wild-type D39 and D39 $\Delta$ lyt $R$ strains were grown in normal $\mathrm{C}+\mathrm{Y}$ medium. For the complemented strain, cells were grown in $\mathrm{C}+\mathrm{Y}$ medium supplemented with $0.15 \mathrm{mM} \mathrm{ZnCl}_{2}$. Bacteria were harvested, resuspended in lysis buffer, and then subjected to immunoblotting assays using rabbit anti-serotype 2 antiserum. GADPH was used as an internal control. Dot blot results showed that, compared with wild-type D39 strain, D39 $\Delta l y t R$ mutant was reduced in the amount of CPS in the bacterial lysates (Figure 5A). Surprisingly, more CPS in the bacterial lysates was observed in complemented strain and $\Delta l y t R$ mutant supplemented with LytR protein in 


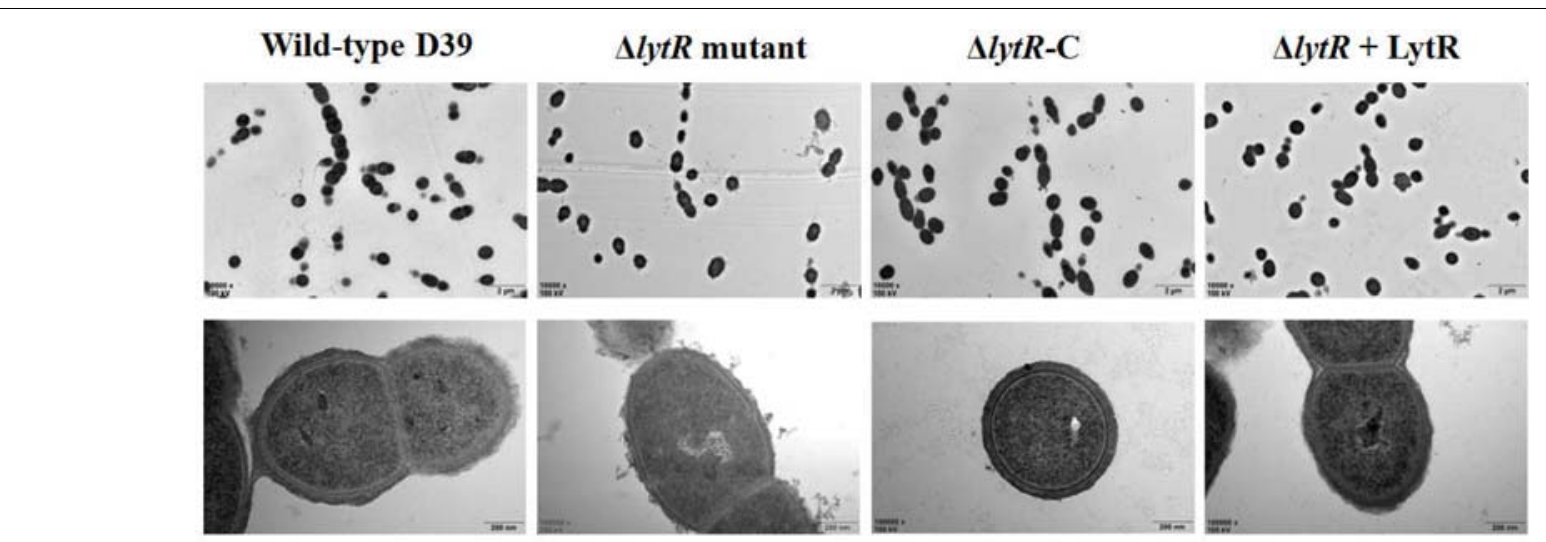

FIGURE 4 | Electron micrographs of strains. Representative figures are shown. $\Delta / y t R$ mutant: D39 $\Delta / y t R ; \Delta / y t R-C:$ complemented strain; $\Delta / y t R+$ LytR: mutant grown in the presence of $500 \mathrm{ng} \Delta \mathrm{TM}$-LytR protein.

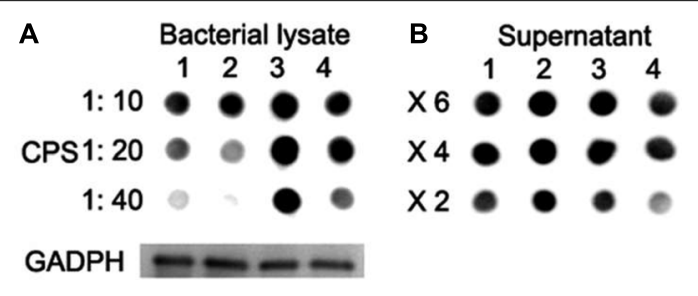

FIGURE 5 | CPS quantification with Dot blot assays. (A) Lane 1: wild-type D39; lane 2: $\Delta / y t R$ mutant; lane 3: complemented strain; lane 4: $\Delta / y t R$ mutant grown in $\mathrm{C}+\mathrm{Y}$ medium plus $500 \mathrm{ng}$ LytR protein. 1:10, 1:20 and 1:40 indicate the diluted fold of samples. (B) X6, X4 and X2 indicate the concentration fold of the culture medium. GADPH was used as loading control. Three independent experiments were performed and representative figures were presented.

comparison with the wild-type strain. We also analyzed the amount of CPS in the culture medium, and it was found that more CPS could be detected in the culture supernatant of the $\Delta l y t R$ mutant (Figure 5B). These results confirmed the results from TEM analysis, and further suggested the direct involvement of LytR in anchoring the CPS to the cell wall.

\section{LytR Was Involved in the Attachment of Pneumococcal TAs}

It has been indicated that the pneumococcal TAs were related with the growth of pneumococci (Liu et al., 2017). As shown in Figure 3B, the $\Delta l y t R$ mutant was impaired in growth. We therefore hypothesized that the $l y t R$ gene may be involved in the attachment of TAs to bacterial surface. Western blot was used to analyze the amount of TAs in whole-cell lysates using monoclonal antibody against phosphorylcholine of TAs (TEPC$15)$. Wild-type D39 and the $\Delta l y t R$ mutant were grown in normal $\mathrm{C}+\mathrm{Y}$ medium. Cells of complemented strain were grown in the presence of $0.15 \mathrm{mM} \mathrm{ZnCl}_{2}$. The amounts of TAs in bacterial lysates and supernatant of the $\Delta l y t R$ mutant grown in the $\mathrm{C}+\mathrm{Y}$ medium supplemented with $500 \mathrm{ng}$ LytR were also determined. Whole-cell lysates were separated with SDS-PAGE, and TAs were probed with TEPC-15. As shown in Figure 6A, compared to wild-type strain, the $\Delta l y t R$ mutant showed smaller TA bands and the density of the bands is weaker. We hypothesized that TAs were released into the culture medium. We therefore tested the amounts of TAs in the culture supernatant using Western blot and Dot blot. It was found that, compared to wildtype and the complemented strains, more TAs in the culture medium of the $\Delta l y t R$ mutant could be detected. To confirm the changes observed in Western blot assays, the amount of TAs was determined by ELISA. In line with the Western blot results, the amounts of TAs in the lyt $R$ mutants were about $2 / 3$ of the levels of the wild-type D39 (Figure 6B), and significant more TAs were detected in the culture medium of the lytR mutant. Complementation with LytR restored the amount of TAs.

\section{Role of LytR in Resistance to Phagocytosis}

Given the critical role of LytR in biosynthesis of both CPS and TAs, we next tested the impairment of LytR mutation in bacterial virulence using in vitro anti-phagocytosis assays. Wild-type D39, the lytR mutant, complemented strains were incubated with macrohages cells for $30 \mathrm{~min}$, and cell lysates were plated onto the blood agar plates for bacterial counting. As shown in Figure 7, the lytR mutant was very sensitive to the killing by macrophages, showing five- to sixfold reduction in survival of the lyt $R$ mutant in relative to that of the wild-type strain (Figure 7). The addition of Ply in the culture medium of the lytR mutant failed to improve the survival, while the addition of LytR significantly improved the survival of the lytR mutant. This observation suggested that the LytR protein is critical for the full virulence of pneumococci.

\section{LytR Mutation Attenuated the Pneumococcal Virulence}

To further understand the role of LytR in pneumococal virulence, we assess the contribution of LytR in pneumococcal colonization and systemic virulence. To determine whether LytR contribute to pneumococcal colonization at the respiratory tracts, groups 


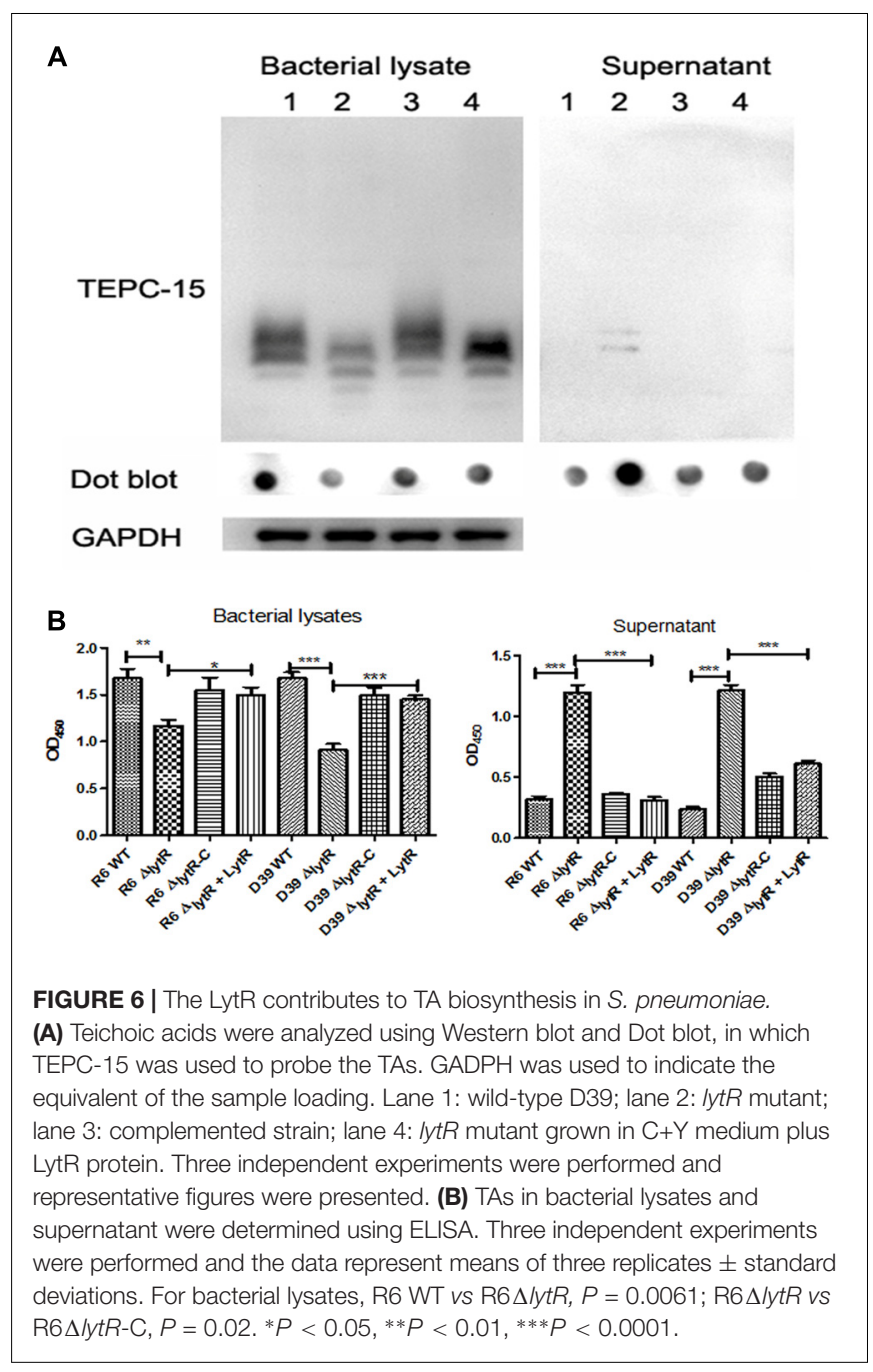

of twelve C57BL6 mice were inoculated intranasally with wildtype D39, D39 $\Delta l y t R$, or $\Delta l y t R-\mathrm{C}$ at a dose of $10^{7} \mathrm{CFU}$. Six mice from each group were killed after 24 and $48 \mathrm{~h}$, and the numbers of bacteria recovered from nasopharynx were determined and compared (Figure 8A). Compared to wild-type D39 strain, the $\Delta l y t R$ mutant showed a significant decrease in numbers of pneumococci colonizing the nasopharynx at both $24 \mathrm{~h}$ and $48 \mathrm{~h}$. Compared with the wild-type D39 strain, a significant decrease in bacterial load of the nasopharynx was also observed at both $24 \mathrm{~h}$ and $48 \mathrm{~h}$ postinoculation for the $\Delta l y t R-\mathrm{C}$ strain.

Furthermore, $24 \mathrm{~h}$ and $48 \mathrm{~h}$ after intranasal infection with S. pneumoniae D39, lung tissues were removed and subjected to histological examination using hematoxylineeosin staining (Figure 8B). The results showed that, mice challenged with the lytR mutant only had a mild inflammation in their lungs. Inflammation was readily apparent in mice challenged with the wild-type D39, with obvious infiltration of neutrophils and monocytes, and even severe necrotic debris in lungs. Inflammation was apparent in mice challenged with the complemented strain at $24 \mathrm{~h}$, but was readily absorbed at $48 \mathrm{~h}$.

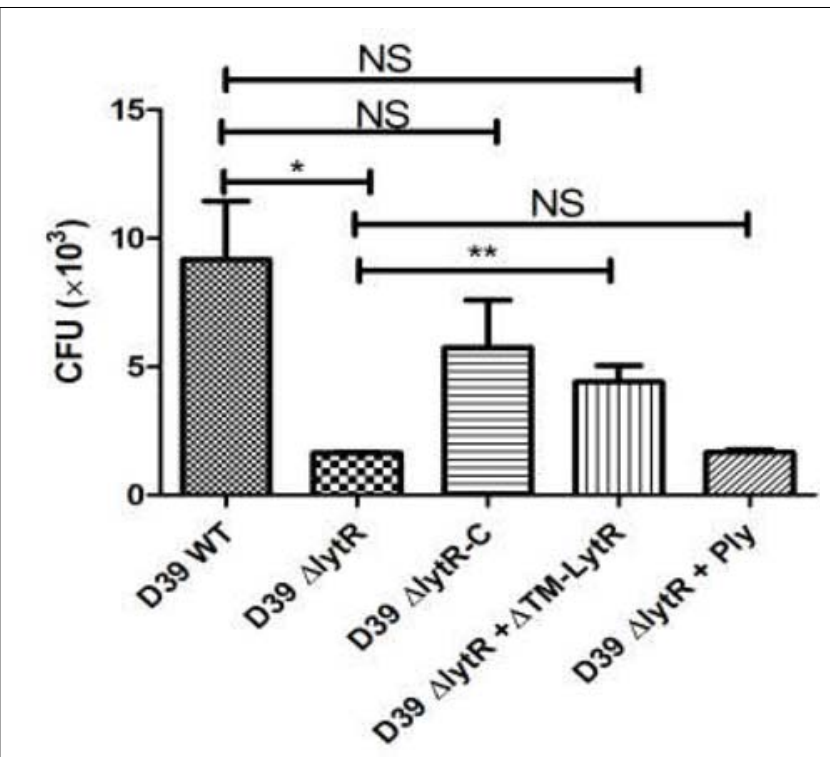

FIGURE 7 | Pneumococcal LytR contributed to resistance to phagocytosis. D39 $\Delta / y t R+\Delta T M-L y t R:$ D39 $\Delta / y t R$ cells were grown in the presence of $500 \mathrm{ng}$ LytR; D39 $\Delta / y t R+$ Ply: D39 $\Delta / y t R$ cells were grown in the presence of $500 \mathrm{ng}$ Ply. D39 WT vs D39 $\Delta / y t R, P=0.016$; D39 $\Delta / y t R+$ LytR vs D39 $\Delta / y t R$, $P=0.0052 ;{ }^{*} P<0.05,{ }^{* *} P<0.01$; NS: not significant. Three independent experiments were performed and the data represent means of three replicates \pm standard deviations.

Obvious inflammation was not observed in mice challenged with normal saline water (NS) at both $24 \mathrm{~h}$ and $48 \mathrm{~h}$.

To investigate the role of LytR in systemic infection, groups of twelve C57BL6 mice were challenged intranasally with wild-type D39, D39 $\Delta l y t R$ or $\Delta l y t R-C$ at a dose of $10^{7} \mathrm{CFU}$. Mice receiving NS was used as negative control. As shown in Figure 8C, all mice challenged with D39 $\Delta l y t R$ and NS survived at the end of the observation period, and $83.3 \%(10 / 12)$ of the mice died from the infection with wild-type D39. The differences in survival rate were significant among the groups, suggesting that LytR is important for full virulence of pneumococci.

\section{DISCUSSION}

Here, by inactivating $l y t R$ gene alone, we present evidence that the $S$. pneumoniae LytR protein is essential for growth and we suggest that it is responsible for attachment of both CPS and TAs to the cell wall. In addition, in line with its function, we observed a significant reduction in pneumococcal virulence when LytR was inactivated. Thus, the present study increases our knowledge concerning the biosynthesis of pneumococcal cell wall.

Gale et al. (2017) has reported that B. subtilis LCP proteins, by acting as a ligase, attached WTAs to peptidoglycan in vitro. By comparing the sequence identity, it was found that $S$. pneumoniae LytR (SPD_1741) showed 32.5\% identity with the B. subtilis DJ97_1211 in full-length amino acid sequence (Supplementary Figure S2). In this study, it was impressive that the D39 $\Delta$ lytR mutant was impaired in both the retention of CPS and TAs. 

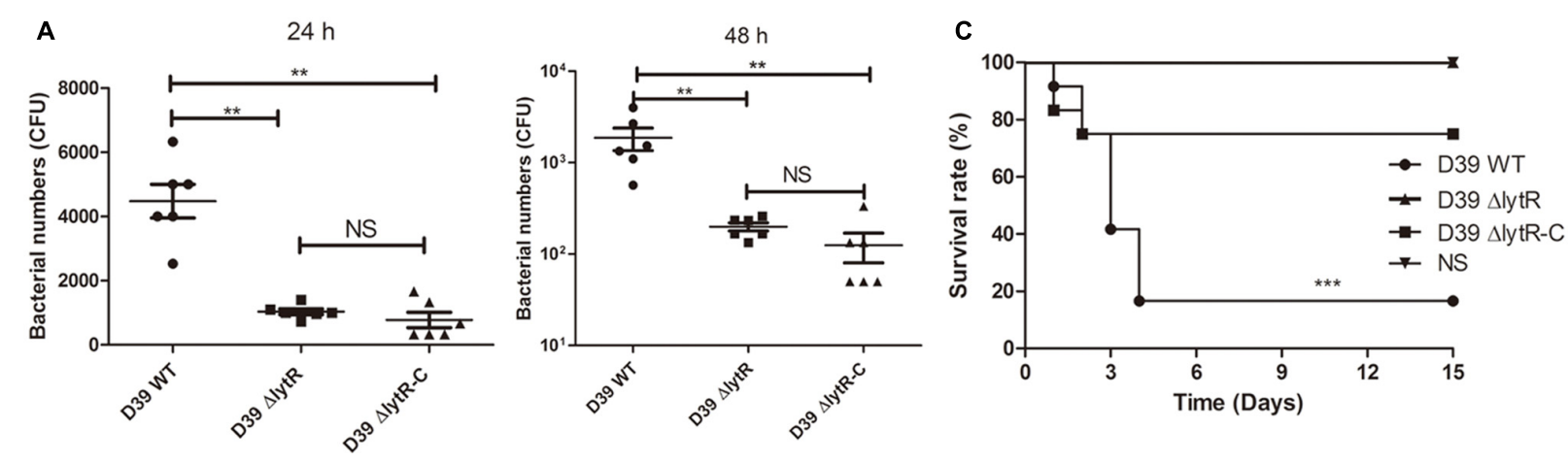

B wild-type D39
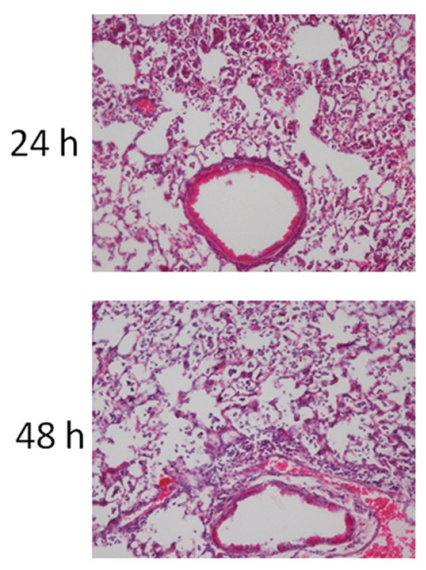

$\Delta l y t R$
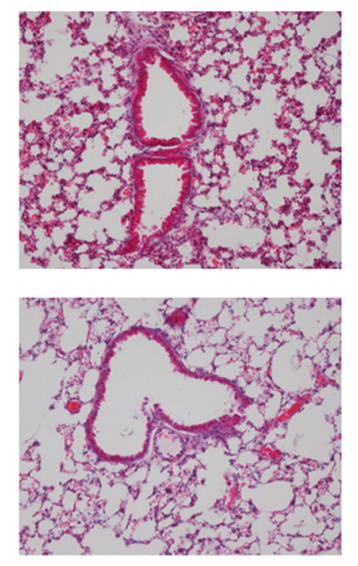

$\triangle l y t R-C$
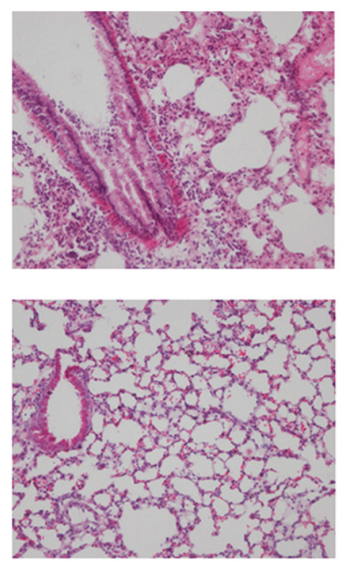

NS
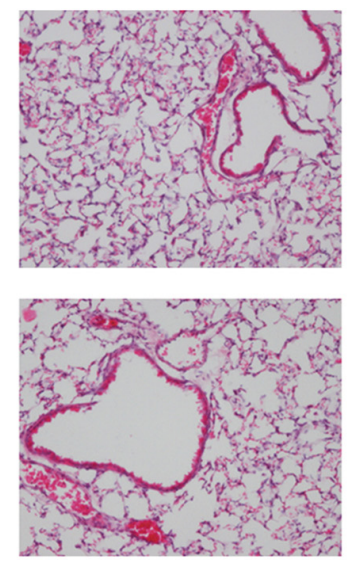

FIGURE 8 | The role of LytR in nasopharyngeal colonization and systemic virulence. (A) C57BL6 mice were challenged with $10^{7}$ CFU S. pneumoniae D39 and pneumococci were recovered from the nasopharynx 24 or $48 \mathrm{~h}$ after intranasal infection of the mice. The graphs show bacterial numbers (CFUs) recovered from nasopharynx of six mice. Data are the mean (SD). ${ }^{*} P<0.01$; Statistical deference was determined by unpaired two-tailed Student's $t$-test. (B) HE staining images of lung tissues of mice $24 \mathrm{~h}$ and $48 \mathrm{~h}$ after challenge with the indicated strains with the same challenge dose as the colonization experiments. $\Delta / y t R$-C: complemented strain. NS: Normal saline water. (C) C57BL6 mice were infected intranasally with $10^{7}$ cells of bacteria. Survival of the mice was recorded, and the difference in survival rates was analyzed using log-rank. $* * * P<0.0001$.

Exogenous addition of LytR protein restored the attachment of both CPS and TAs to the bacterial cells. In Staphylococcus aureus, the phosphosugar transferase activity of LCP proteins has been presented by showing a direct enzymatic role in ligation of WTA to peptidoglycan substrates (Schaefer et al., 2017). Thus, it is likely that the LytR may function as a ligase in the retention of both CPS and TAs to the cell wall in S. pneumoniae.

Knocking down Cps2A did not impair the growth profile in S. pneumoniae D39 (Eberhardt et al., 2012), suggesting that the loss of CPS alone may not influence the division or autolysis and hence the growth rate. This study demonstrated that the lytR mutant grew very slowly and the stationary phase was missing (Figure 2). On the basis of the growth results, the phenotypes of lyt $R$ mutant are consistent with the typical growth defect caused by repression of genes in TA biosynthesis (Liu et al., 2017). This may be explained by the observation that $l y t R$ was involved in the biosynthesis of TAs (Figure 6). It is known that TA was important for the biological roles in S. pneumoniae; however, the attachment to the cell remains elusive. Our previous results suggested that raf $X$ may function as a ligase attaching the TA precursors to the cell wall; However, rafX inactivation did not lead to complete loss of the TAs in S. pneumoniae strains (Wu et al., 2014), indicating the involvement of other enzymes in this process. In this study, deletion of the lytR gene also impaired the attachment of TAs to bacteria, suggesting the enzymatic effect of LytR in this process. Of note, the size of TAs was different in the lytR mutant compared to wild-type and the complemented strains (Figure 6). One possible explanation for this may be relevant to the less amounts of TAs to cell wall which may lead to faster migration of TAs in the lytR mutant than that of the wild-type strain. Another possible reason is that LytR might lead to modification in TAs which needs to be further investigated. Nevertheless, our findings strengthen our knowledge on pneumococcal TA biosynthesis.

Contrary with previous studies showing that LytR could not be inactivated alone in S. pneumoniae D39 (Eberhardt et al., 2012), the present study replaced the lytR gene with erm cassette. 
The deletion of $l y t R$ gene was proved to be a nonpolar event verified by reverse transcription (RT)-PCR and sequencing. In addition, the D39 $\Delta$ lyt $R$ mutant did not display very obvious morphological and division abnormity as did the R6 $\operatorname{lyt} R$ mutant (Johnsborg and Havarstein, 2009). The discrepancy in obtaining the lytR mutant may be due to the different genetic background pneumococcal strains used, or may be caused by the difference in selection marker where kanamycin was used as a selection marker for gene deletion in their study.

In this study, we observed an increased amount of CPS in the culture supernatant of the lytR mutant, which was an indication of unbound portions due to the function loss of LytR. This is true because exogenous addition of LytR reduced the amount of CPS in culture supernatant and more CPS was observed in bacterial cells (Figure 5). It was found that the complemented strain (ectopically complemented with the plasmid lytR-pJWV25) produced more CPS than did the wildtype D39 as shown in Figure 4. We therefore examined the level of LytR in the complemented strain and found that a higher level of LytR was produced in complemented strain than the wildtype D39 (Supplementary Figure S3). This observation highlights that the ligation of CPS to the cell wall may be dependent on the LytR concentrations, which is consistent with the enzyme reaction feature.

It has been reported that the inactivation of $c p s 2 A$ alone only led to $29 \%$ reduction in the amount of surface CPS. In this study, it was found that about half of the capsule was missing on the surface of bacterial cells in the lytR mutant (Figures 4, 5). It was surprising that the $l y t R$ deficient mutant had equal amount of CPS as the $c P s A$ lytR double mutant shown in a previous study (Eberhardt et al., 2012). It seems that LytR was more important than Cps2A in the retention of CPS to the cell wall.

It is of note that despite we named $\Delta l y t R-C$ as the complemented strain, as shown in Figures 6, 7, the phenotype of $\Delta l y t R-C$ was not restored to the wild-type levels. This is because that the complemented effect relays on the concentrations of $\mathrm{Zn}^{2+}$ (Eberhardt et al., 2009). Thus, when complemented strain was inoculated into the animals, the concentrations of $\mathrm{Zn}^{2+}$ may be not sufficient to support a continuous expression of LytR and hence may lead to the attenuation of the complemented strain, and this may be used to explain why complemented strain was not as virulent as the wild-type in the infection experiments. Although the $\Delta l y t R-\mathrm{C}$ strain failed to kill animals as the wild type D39 in animal infection experiments, the results in Figure 7 did not show significant difference in resistance to

\section{REFERENCES}

Arrecubieta, C., Lopez, R., and Garcia, E. (1994). Molecular characterization of cap3A, a gene from the operon required for the synthesis of the capsule of Streptococcus pneumoniae type 3: sequencing of mutations responsible for the unencapsulated phenotype and localization of the capsular cluster on the pneumococcal chromosome. J. Bacteriol. 176, 6375-6383. doi: 10.1128/jb.176.20.6375-6383. 1994

Cao, J., Xu, F., Lin, S., Song, Z., Zhang, L., Luo, P., et al. (2014). IL-27 controls sepsis-induced impairment of lung antibacterial host defence. Thorax 69, 926-937. doi: 10.1136/thoraxjnl-2014-205777 killing by phagocytosis among wild type D39, D39 $\Delta l y t R-\mathrm{C}$ and $\mathrm{D} 39 \Delta l y t R+\Delta \mathrm{TM}-\mathrm{LytR}$ suggesting the complementation of LytR and the contribution of LytR in bacterial virulence.

\section{CONCLUSION}

Our results provide strong evidence that pneumoccal LyR is critical for some basic cellular functions and important for pneumococcal virulence. Since LytR is a membrane protein and is surface exposed, we anticipate that pneumococcal LytR can be a novel drug target to cure pneumococcal infection.

\section{AUTHOR CONTRIBUTIONS}

$\mathrm{YY}, \mathrm{XZ}$, and KW conceived and designed the experiments. WY, $\mathrm{JZ}$, and ZS performed the experiments. WY, YY, XZ, and KW analyzed the data. YY, XZ, and KW contributed the reagents, materials, and analysis tools. WY, XZ, and KW wrote the paper. All authors read and approved the final manuscript.

\section{FUNDING}

This work was supported by Projects of the National Natural Science Foundation of China (Nos. 31600115 and 81772153), "15851 talent project" provided by Zunyi City in 2015 \& 2016, Talent project provided by Guizhou Province (Zunshi Kehe Rencai 2017-32), and Launch funding for M.D. provided by the first people's hospital of Zunyi.

\section{ACKNOWLEDGMENTS}

We thank Jan-Willem Veening for providing plasmids pJWV25. We thank Xiaogang Liao, Hong Wang, and Jingchuan Fan for electron microscopy work.

\section{SUPPLEMENTARY MATERIAL}

The Supplementary Material for this article can be found online at: https://www.frontiersin.org/articles/10.3389/fmicb. 2018.01199/full\#supplementary-material

Chan, Y. G., Frankel, M. B., Dengler, V., Schneewind, O., and Missiakas, D. (2013). Staphylococcus aureus mutants lacking the LytR-CpsA-Psr family of enzymes release cell wall teichoic acids into the extracellular medium. J. Bacteriol. 195, 4650-4659. doi: 10.1128/JB.00544-13

Chan, Y. G., Kim, H. K., Schneewind, O., and Missiakas, D. (2014). The capsular polysaccharide of Staphylococcus aureus is attached to peptidoglycan by the LytR-CpsA-Psr (LCP) family of enzymes. J. Biol. Chem. 289, 15680-15690. doi: 10.1074/jbc.M114.567669

Denapaite, D., Bruckner, R., Hakenbeck, R., and Vollmer, W. (2012). Biosynthesis of teichoic acids in Streptococcus pneumoniae and closely related species: lessons from genomes. Microb. Drug Resist. 18, 344-358. doi: 10.1089/mdr.2012. 0026 
Eberhardt, A., Hoyland, C. N., Vollmer, D., Bisle, S., Cleverley, R. M., Johnsborg, O., et al. (2012). Attachment of capsular polysaccharide to the cell wall in Streptococcus pneumoniae. Microb. Drug Resist. 18, 240-255. doi: 10.1089/mdr.2011.0232

Eberhardt, A., Wu, L. J., Errington, J., Vollmer, W., and Veening, J. W. (2009). Cellular localization of choline-utilization proteins in Streptococcus pneumoniae using novel fluorescent reporter systems. Mol. Microbiol. 74, 395-408. doi: 10.1111/j.1365-2958.2009.06872.x

Gale, R. T., Li, F. K. K., Sun, T., Strynadka, N. C. J., and Brown, E. D. (2017). B. subtilis LytR-CpsA-Psr Enzymes transfer wall teichoic acids from authentic lipid-linked substrates to mature peptidoglycan In Vitro. Cell Chem. Biol. 24, 1537.e4-1546.e4. doi: 10.1016/j.chembiol.2017.09.006

Hammerschmidt, S., Wolff, S., Hocke, A., Rosseau, S., Muller, E., and Rohde, M. (2005). Illustration of pneumococcal polysaccharide capsule during adherence and invasion of epithelial cells. Infect. Immun. 73, 4653-4667. doi: 10.1128/IAI. 73.8.4653-4667.2005

Hardy, G. G., Magee, A. D., Ventura, C. L., Caimano, M. J., and Yother, J. (2001). Essential role for cellular phosphoglucomutase in virulence of type 3 Streptococcus pneumoniae. Infect. Immun. 69, 2309-2317. doi: 10.1128/IAI.69. 4.2309-2317.2001

Hoogerwerf, J. J., Leendertse, M., Wieland, C. W., De Vos, A. F., De Boer, J. D., Florquin, S., et al. (2011). Loss of suppression of tumorigenicity 2 (ST2) gene reverses sepsis-induced inhibition of lung host defense in mice. Am. J. Respir. Crit. Care Med. 183, 932-940. doi: 10.1164/rccm.201006-0934OC

Hyams, C., Camberlein, E., Cohen, J. M., Bax, K., and Brown, J. S. (2010). The Streptococcus pneumoniae capsule inhibits complement activity and neutrophil phagocytosis by multiple mechanisms. Infect. Immun. 78, 704-715. doi: 10.1128/IAI.00881-09

Johnsborg, O., and Havarstein, L. S. (2009). Pneumococcal LytR, a protein from the LytR-CpsA-Psr family, is essential for normal septum formation in Streptococcus pneumoniae. J. Bacteriol. 191, 5859-5864. doi: 10.1128/JB. 00724-09

Kawai, Y., Marles-Wright, J., Cleverley, R. M., Emmins, R., Ishikawa, S., Kuwano, M., et al. (2011). A widespread family of bacterial cell wall assembly proteins. EMBO J. 30, 4931-4941. doi: 10.1038/emboj.2011.358

Kodali, V., Littke, M. H., Tilton, S. C., Teeguarden, J. G., Shi, L., Frevert, C. W., et al. (2013). Dysregulation of macrophage activation profiles by engineered nanoparticles. ACS Nano 7, 6997-7010. doi: 10.1021/nn402145t

Liszewski Zilla, M., Chan, Y. G. Y., Lunderberg, J. M., Schneewind, O., Missiakas, D., and Christie, P. J. (2015). LytR-CpsA-Psr enzymes as determinants of Bacillus anthracis secondary cell wall polysaccharide assembly. J. Bacteriol. 197, 343-353. doi: 10.1128/JB.02364-14

Liu, X., Gallay, C., Kjos, M., Domenech, A., Slager, J., Van Kessel, S. P., et al. (2017). High-throughput CRISPRi phenotyping identifies new essential genes in Streptococcus pneumoniae. Mol. Syst. Biol. 13:931. doi: 10.15252/msb.20167449

Llull, D., Munoz, R., Lopez, R., and Garcia, E. (1999). A single gene (tts) located outside the cap locus directs the formation of Streptococcus pneumoniae type 37 capsular polysaccharide. Type 37 Pneumococci are natural, genetically binary strains. J. Exp. Med. 190, 241-251. doi: 10.1084/jem.190.2.241

Mollerach, M., Lopez, R., and Garcia, E. (1998). Characterization of the galU gene of Streptococcus pneumoniae encoding a uridine diphosphoglucose pyrophosphorylase: a gene essential for capsular polysaccharide biosynthesis. J. Exp. Med. 188, 2047-2056. doi: 10.1084/jem.188.11.2047

Schaefer, K., Matano, L. M., Qiao, Y., Kahne, D., and Walker, S. (2017). In vitro reconstitution demonstrates the cell wall ligase activity of LCP proteins. Nat. Chem. Biol. 13, 396-401. doi: 10.1038/nchembio.2302

Sharif, O., Matt, U., Saluzzo, S., Lakovits, K., Haslinger, I., Furtner, T., et al. (2013). The scavenger receptor CD36 downmodulates the early inflammatory response while enhancing bacterial phagocytosis during pneumococcal pneumonia. J. Immunol. 190, 5640-5648. doi: 10.4049/jimmunol.1202270

Skov Sorensen, U. B., Blom, J., Birch-Andersen, A., and Henrichsen, J. (1988). Ultrastructural localization of capsules, cell wall polysaccharide, cell wall proteins, and $\mathrm{F}$ antigen in pneumococci. Infect. Immun. 56, 1890-1896.

Weidenmaier, C., and Peschel, A. (2008). Teichoic acids and related cell-wall glycopolymers in Gram-positive physiology and host interactions. Nat. Rev. Microbiol. 6, 276-287. doi: 10.1038/nrmicro1861

Wu, K., Huang, J., Zhang, Y., Xu, W., Xu, H., Wang, L., et al. (2014). A novel protein, RafX, is important for common cell wall polysaccharide biosynthesis in Streptococcus pneumoniae: implications for bacterial virulence. J. Bacteriol. 196, 3324-3334. doi: 10.1128/JB.01696-14

Wu, K., Xu, H., Zheng, Y., Wang, L., Zhang, X., and Yin, Y. (2016). CpsR, a GntR family regulator, transcriptionally regulates capsular polysaccharide biosynthesis and governs bacterial virulence in Streptococcus pneumoniae. Sci. Rep. 6:29255. doi: 10.1038/srep29255

Wu, K., Zhang, X., Shi, J., Li, N., Li, D., Luo, M., et al. (2010). Immunization with a combination of three pneumococcal proteins confers additive and broad protection against Streptococcus pneumoniae Infections in Mice. Infect. Immun. 78, 1276-1283. doi: 10.1128/IAI.00473-09

Zheng, Y., Zhang, X., Wang, X., Wang, L., Zhang, J., and Yin, Y. (2017). ComE, an essential response regulator, negatively regulates the expression of the capsular polysaccharide locus and attenuates the bacterial virulence in Streptococcus pneumoniae. Front. Microbiol. 8:277. doi: 10.3389/fmicb.2017.00277

Conflict of Interest Statement: The authors declare that the research was conducted in the absence of any commercial or financial relationships that could be construed as a potential conflict of interest.

Copyright (C) 2018 Ye, Zhang, Shu, Yin, Zhang and Wu. This is an open-access article distributed under the terms of the Creative Commons Attribution License (CC BY). The use, distribution or reproduction in other forums is permitted, provided the original author(s) and the copyright owner are credited and that the original publication in this journal is cited, in accordance with accepted academic practice. No use, distribution or reproduction is permitted which does not comply with these terms. 